\title{
MANY-EXCITON COMPLEXES IN SELF-ASSEMBLED QUANTUM DOTS
}

\author{
A. WóJS ${ }^{a}$, P. HAWRYLAK ${ }^{b}$ AND L. JACAK ${ }^{a}$ \\ anstitute of Physics, Technical University of Wrocław \\ Wybrzeże Wyspiańskiego 27, 50-370 Wroclaw, Poland \\ ${ }^{b}$ Institute for Microstructural Sciences, National Research Council of Canada \\ Ottawa, K1A 0R6, Canada
}

The system of interacting electrons and holes confined in a lens-shaped InGaAs self-assembled dot is studied using exact diagonalization techniques. The single-particle energy spectrum of self-assembled dot is well approximated by that of a quasi-two-dimensional atom with parabolic lateral confinement. The electronic shell structure of self-assembled dot is responsible for a remarkable dependence of the absorption/emission spectrum on the number of excitons. This is explained in terms of hidden symmetries leading to a formation of coherent many-exciton states of weakly interacting excitons and bi-excitons.

PACS numbers: $73.20 . \mathrm{Dx}, 71.45 . \mathrm{Gm}, 78.66 . \mathrm{Fd}$

It has been recently demonstrated [1] that an epitaxial growth of strained InAs layers on GaAs is unstable and leads to a spontaneous formation of InAs-rich quasi-2D islands. The actual shape of these quasi-0D objects depends on the growth parameters and different forms have been reported [1-3]. We concentrate here on a class of lens-shaped InGaAs self-assembled dots (SAD's) investigated recently by single electron capacitance (SECS), photoluminescence (PL), and far-infrared (FIR) spectroscopies [1,4-6]. The lens-shaped InGaAs SAD is formed on a InGaAs wetting layer (WL) of thickness $t$, grown on GaAs. The SAD is modeled as a part of a sphere with fixed height $h$ and radius at the base $s$ (see inset in Fig. 1). The structure is overgrown with GaAs. The discontinuities in the conduction and valence band energies between the $\mathrm{SAD} / \mathrm{WL}$ material and the surrounding $\mathrm{GaAs}$ lead to the confinement of conduction electrons and valence holes in the InGaAs layer. Both electrons and holes are further localized in the area of the dot due to the effectively increased thickness of the WL, i.e. the effectively decreased energy of the lowest confined subband in the WL. The detailed calculation [7] shows that the resulting effective lateral potential in the SAD is well approximated by a parabola: $\frac{1}{2} m^{*} \omega_{0}^{2} r^{2}$. Here $m^{*}$ is the effective mass of an electron and $\omega_{0}$ is the 
spacing of energy levels. The exact energy levels, corresponding both to the states bound in a dot and to the WL resonances, form almost degenerate and almost equally spaced shells. The approximate energy levels $E_{n m}=\hbar \omega_{0}(n+m+1)$ and the single-particle states $|n m\rangle$ are those of a two-dimensional harmonic oscillator. This is illustrated in Fig. 1, where we show the numerically calculated electron energy spectrum (measured from the bottom of bulk GaAs continuum) resolved into different angular momentum channels $l=m-n$. The parameters used for calculation correspond to the sample reported by Raymond et al. [4] ( $s=180 \AA$ and $h=44 \AA$ ), yielding 5 confined shells spaced by $30 \mathrm{meV}$ (denoted here as $s$, $p, d, f$ and $g$ ), i.e. 30 confined states including $\pm l$ and spin degeneracy. For the valence holes the number of confined shells is also 5 , and the intershell separation is $15 \mathrm{meV}$.

The dependence of the electron energy spectrum on the dot size and on the step in the conduction band energy at the $\operatorname{In}_{x} \mathrm{Ga}_{1-x} \mathrm{As} / \mathrm{GaAs}$ interface, controlled by the growth conditions and the indium fraction $x$, respectively, has been discussed elsewhere [7]. It is shown that variation of these parameters does not destroy the characteristic shell structure shown in Fig. 1, but only changes the number of confined shells and their separation.

We now turn to the effect of electron-electron (ee), hole-hole $(\mathrm{hh})$ and electron-hole (eh) interactions on the optical properties of quantum dots. Using a composite index $i=[n, m]$, the Hamiltonian of the interacting electron-hole system may be written in a compact form

$$
\begin{aligned}
H= & \sum_{i \sigma} E_{i}^{\mathrm{e}} c_{i \sigma^{\prime}}^{+} c_{i \sigma}+\sum_{i \sigma} E_{i}^{\mathrm{h}} h_{i \sigma}^{+} h_{i \sigma}-\sum_{i j k l \sigma \sigma^{\prime}}\left\langle i j\left|v_{\mathrm{eh}}\right| k l\right\rangle c_{i \sigma}^{+} h_{j \sigma^{\prime}}^{+} h_{k \sigma^{\prime}} c_{l \sigma} \\
& +\frac{1}{2} \sum_{i j k l \sigma \sigma^{\prime}}\left\langle i j\left|v_{\mathrm{ee}}\right| k l\right\rangle c_{i \sigma}^{+} c_{j \sigma^{\prime}}^{+} c_{k \sigma^{\prime}} c_{l \sigma} \\
& +\frac{1}{2} \sum_{i j k l \sigma \sigma^{\prime}}\left\langle i j\left|v_{\mathrm{hh}}\right| k l\right\rangle h_{i \sigma}^{+} h_{j \sigma^{\prime}}^{+} h_{k \sigma^{\prime}} h_{l \sigma} .
\end{aligned}
$$

Operators $c_{i \sigma}^{+}\left(c_{i \sigma}\right), h_{i \sigma}^{+}\left(h_{i \sigma}\right)$ create (annihilate) an electron/hole with the spin $\sigma$ on the orbital $i$ with the single-particle energy $E_{i}$, and $\langle i j|v| k l\rangle$ are the two-body Coulomb matrix elements [8].

The Hilbert space of $N$ electron-hole pair ( $N$-exciton) states is spanned by: $\widehat{A}\left(\prod_{i=1}^{N} c_{j_{i} \sigma_{i}}^{+}\right)\left(\prod_{i=1}^{N} h_{k_{i} \sigma_{i}}^{+}\right)|v a c\rangle$ - anti-symmetrized products of the single-particle states. Each state is classified by a total angular momentum $R_{\text {tot }}$ and a total spin $S_{\text {tot }}^{z}$. The Hilbert space can be divided into subspaces labelled by $R_{\text {tot }}$ and $S_{\text {tot }}^{z}$, block-diagonalizing Hamiltonian (1). We diagonalize Hamiltonian in the optically active subspace: $R_{\mathrm{tot}}=0, S_{\mathrm{tot}}^{z}=0$, for up to 6 excitons. A combination of the numerical diagonalization within partially filled shells and the Hartree-Fock approximation extended calculations for up to 20 excitons [9].

Interband optical properties of the SAD are described by the angular-momentum-like algebra of an interband optical polarization operator $\boldsymbol{P}$. The physical meaning of the three components of $\boldsymbol{P}$ is very transparent: $P^{+}=\sum_{i} c_{i}^{+} h_{i}^{+}$ $\left(P^{-}=\sum_{i} h_{i} c_{i}\right)$ coherently creates (annihilates) an electron-hole pair over all orbitals $i$, and $P_{z}=\sum_{i}\left(c_{i}^{+} c_{i}+h_{i}^{+} h_{i}-1\right)$ measures the number of created pairs. The 


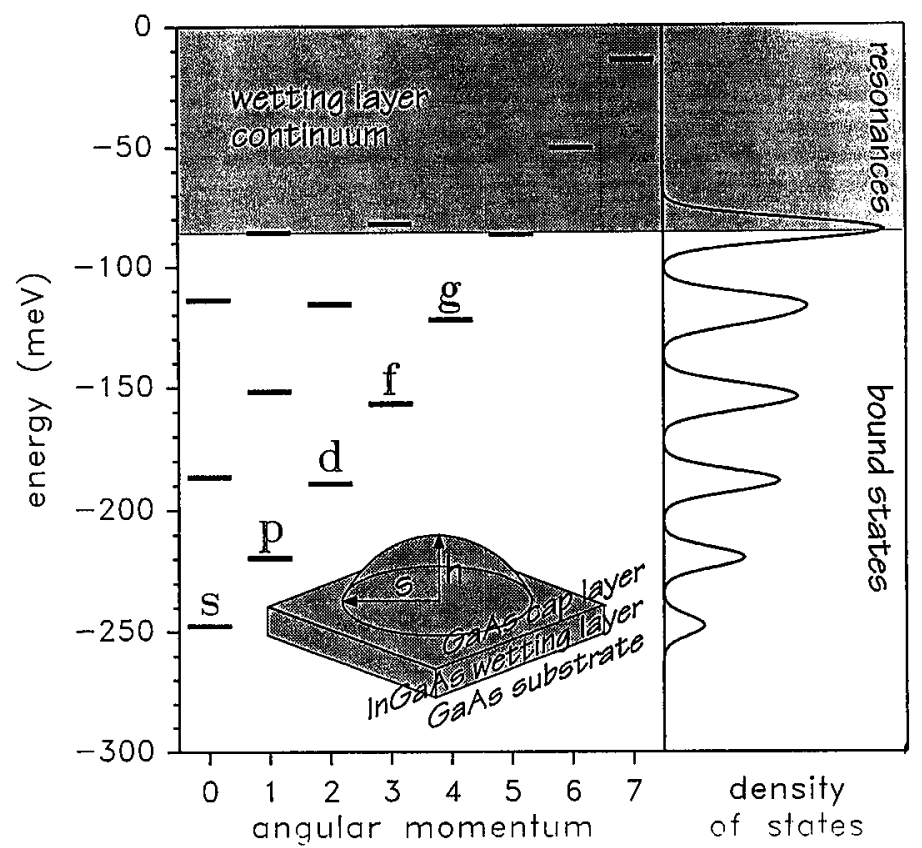

Fig. 1. Left: electron energy spectrum of bound states and wetting-layer resonances in the InGaAs/GaAs SAD; average intershell spacing is $30 \mathrm{meV}$; inset: schematic of the SAD modeled as a part of the sphere on the narrow wetting layer. Right: the approximate density of states (individual levels broadened with Gaussians).

two distinct spin polarizations $\sigma$, created by two different circular polarizations of light [8], are described by the two polarization operators $\boldsymbol{P}_{\sigma}$.

We now turn to the discussion of numerical results. Here we concentrate on the $N$-exciton addition spectrum as a function of the number of excitons already present in the SAD, shown in Fig. 2. The addition spectrum represents the absorption spectrum (or equivalently the optical density of states, DOS) of an exciton in the presence of $N-1$ excitons. We assume that excitons are added using linearly polarized light. The absorption spectrum for $N=1$ consists of a series of peaks corresponding to electron/hole degenerate shells, which resembles the DOS of the dot. The actual structure can be traced back to the coupling of the center-of-mass and relative motion of the exciton. For $N=2$ the absorption spectrum is basically the DOS of a bi-exciton (XX). The ground state (GS) addition energy of $\mathrm{XX}$ is lower than that of $\mathrm{X}$ by $5 \mathrm{meV}$, and there is a significant modification of the oscillator strength due to the blocking of the phase space by an existing exciton. The phase space blocking becomes dramatic for $N=3$, when an exciton is added to a bi-exciton. No spectral weight is present at energies corresponding to the GS energy of X, due to the occupation of the $s$-shell orbital by XX. Surprisingly, the GS addition energy for $N=3-6$ does not appear to depend on $N$. However, a visible effect is the red-shift of the energies of higher peaks (band-gap renormalization). The numerically obtained spectra indicate that within a filled shell, excitons and bi-excitons form a weakly interacting gas. This cancellation of the effects of electron-electron and electron-hole interactions can be understood in terms of hidden symmetries. 


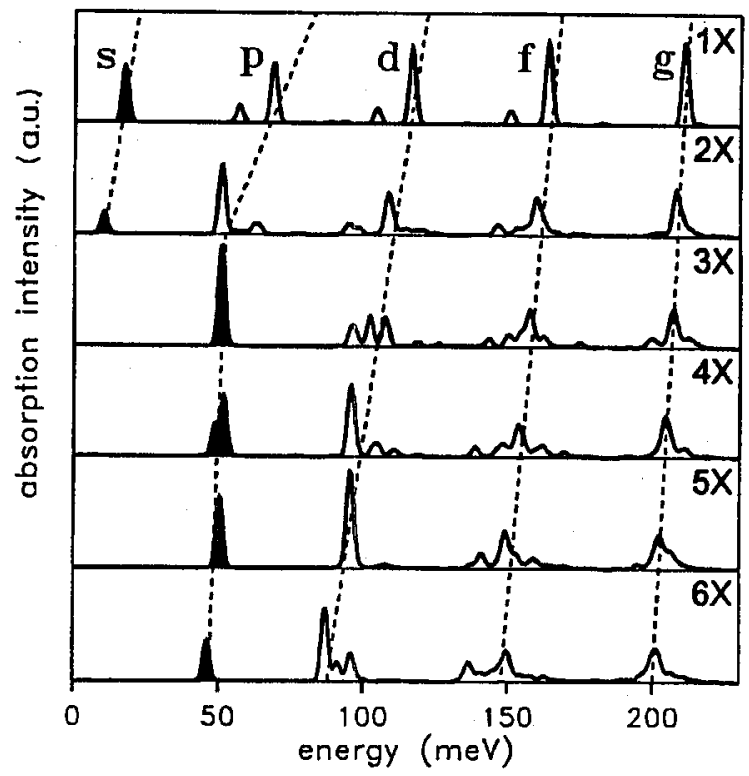

Fig. 2. Absorption spectra of 1-6 excitons in the InGaAs/GaAs SAD; shaded peaks correspond to creation of an exciton within the partially filled shell.

Let us consider a pair of commuting operators: $\left(P^{2}, P_{z}\right)$. While $H$ clearly conserves $P_{z}$, the conservation of $P^{2}$ depends on the commutator between $H$ and $P^{ \pm}$. When two necessary conditions are met: (a) degenerate electron and hole energy levels, (b) equal ee, hh, and eh interactions, the commutator can be written as $\left[H, P^{+}\right]=E_{\mathrm{X}} P^{+}[9,10]$, where $E_{\mathrm{X}}$ is an approximate exciton energy. This implies the commutation between $H$ and $P^{2}$, and the eigenstates of $\left(P^{2}, P_{z}\right)$ are the eigenstates of $H$. The $N$-exciton GS within an $L$-fold shell is obtained by subsequent application of $P^{+}$on vacuum: $\left(P^{+}\right)^{N}|\mathrm{vac}\rangle(N=0,1, \ldots, L)$. We shall call such GS's the coherent many-exciton states (CMES). They correspond to: $P=\frac{1}{2} L$ and $P_{z}=N-\frac{1}{2} L$. Using a convenient analogy between $P$ and the total spin of $L$ spin- $\frac{1}{2}$ particles (mapping: $c^{ \pm} \sim a_{\uparrow}^{ \pm}$and $h^{ \pm} \sim a_{\downarrow}^{\mp}$ ), adding (subtracting) excitons to (from) a shell can be viewed as rotating the polarization vector $\boldsymbol{P}$ without changing its length $P$. The empty (completely filled) shell is represented by $\boldsymbol{P}$ pointing down (up), i.e.: $P_{z}=-(+) \frac{1}{2} L$. The GS energy of a shell is linear in $N$ and the addition/subtraction energy is a constant. Hence, despite strong correlations in the system, the consecutive excitons are added to the shell with the same energy, forming a condensate. What our extensive numerical results show is that the overlap of CMES wave function with the actual GS wave function is $\approx 1$.

In conclusion, we have studied the system of many interacting electrons and holes, confined in a SAD. The SAD can be viewed as an artificial atom, characterized by its electron and hole shell structure. When the SAD is filled with excitons, this shell structure leads to their condensation into the many-exciton coherent state. The spin of electrons and holes leads to the binding of bi-excitons, 
and splitting of the exciton addition energy. The exchange interaction between different shells is mainly responsible for a band-gap renormalization.

The authors acknowledge useful discussions with S. Fafard, S. Raymond, P. Poole, S. Charbonneau, L.G. Rego, J.A. Brum, A.H. MacDonald. One of us (A.W.) acknowledges financial support by the Institute for Microstructural Sciences, NRC Canada.

\section{References}

[1] P.M. Petroff, S.P. Denbaars, Superlattices Microstruct. 15, 15 (1994); for a recent review see Solid State Electron., to be published.

[2] M. Grundmann, N.N. Ledentsov, R. Heitz, L. Eckey, J. Christen, J. Böhrer, D. Bimberg, S.S. Ruvimov, P. Werner, U. Richter, J. Heydenreich, V.M. Ustinov, A.Y. Egorov, A.E. Zhukov, P.S. Kopev, Z.I. Alferov, Phys. Status Solidi B 188, 249 (1995).

[3] J.Y. Marzin, G. Bastard, Solid State Commun. 92, 437 (1994).

[4] S. Raymond, S. Fafard, P.J. Poole, A. Wojjs, P. Hawrylak, S. Charbonneau, D. Leonard, R. Leon, P.M. Petroff, J.L. Merz, to be published.

[5] H. Drexler, D. Leonard, W. Hansen, J.P. Kotthaus, P.M. Petroff, Phys. Rev. Lett. 73, 2252 (1994).

[6] A. Wójs, P. Hawrylak, Phys. Rev. B 53, 10841 (1996).

[7] A. Wójs, P. Hawrylak, S. Fafard, L. Jacak, to be published.

[8] A. Wójs, P. Hawrylak, Phys. Rev. B 51, 10880 (1995).

[9] A. Wójs, P. Hawrylak, to be published.

[10] I.V. Lerner, Yu.E. Lozovik, Zh. Eksp. Teor. Fiz. 80, 1488 (1981) [Sov. Phys. JETP 53, 763 (1981)]; D. Paquet, T.M. Rice, K. Ueda, Phys. Rev. B 32, 5208 (1985); A.H. MacDonald, E.H. Rezayi, Phys. Rev. B 42, 3224 (1990). 